\title{
Partially hydrolysed guar gum ameliorates murine intestinal inflammation in association with modulating luminal microbiota and SCFA
}

\author{
Tomohisa Takagi $^{1 *}$, Yuji Naito ${ }^{1}$, Yasuki Higashimura ${ }^{1}$, Chihiro Ushiroda ${ }^{1}$, Katsura Mizushima ${ }^{1}$, Yuji \\ Ohashi $^{2}$, Zenta Yasukawa ${ }^{3}$, Makoto Ozeki ${ }^{3}$, Makoto Tokunaga ${ }^{3}$, Tsutomu Okubo ${ }^{3}$, Kazuhiro Katada ${ }^{1}$, \\ Kazuhiro Kamada ${ }^{1}$, Kazuhiko Uchiyama ${ }^{1}$, Osamu Handa ${ }^{1}$, Yoshito Itoh ${ }^{1}$ and Toshikazu Yoshikawa ${ }^{1}$ \\ ${ }^{1}$ Department of Molecular Gastroenterology and Hepatology, Graduate School of Medical Science, Kyoto Prefectural University \\ of Medicine, Kyoto, 602-8566, Japan \\ ${ }^{2}$ Laboratory of Food Hygiene, Department of Food Science and Technology, Nippon Veterinary and Life Science University, \\ 1-7-1, Kyonan-cho, Musashino, Tokyo 180-8602, Japan \\ ${ }^{3}$ Nutrition Division, Taiyo Kagaku Co. Ltd, 1-3 Takaramachi, Yokkaichi 510-0844, Japan
}

(Submitted 27 May 2016 - Final revision received 21 July 2016 - Accepted 22 July 2016 - First published online 8 September 2016)

\begin{abstract}
Partially hydrolysed guar gum (PHGG), a water-soluble dietary fibre produced by the controlled partial enzymatic hydrolysis of guar gum beans, has various physiological roles. This study aimed to elucidate the beneficial effects of PHGG on colonic mucosal damage in a murine 2,4,6-trinitrobenzene sulfonic acid (TNBS)-induced colitis model. Acute colitis was induced in male C57BL/6 mice with TNBS after 2 weeks of pre-feeding with PHGG (5\%). The colonic mucosal inflammation was evaluated using macroscopic damage scores, and neutrophil infiltration was assessed by measuring tissue-associated myeloperoxidase (MPO) activity in the colonic mucosa. TNF- $\alpha$ expression in the colonic mucosa was measured by ELISA and real-time PCR. Moreover, the intestinal microbiota and production of SCFA were assessed by real-time PCR and HPLC, respectively. Colonic damage due to TNBS administration was significantly ameliorated by PHGG treatment. Furthermore, PHGG significantly inhibited increases in MPO activity and TNF- $\alpha$ protein and mRNA expression in the colonic mucosa in TNBS-induced colitis. On analysis of intestinal microbiota, we found that the concentration of the Clostridium coccoides group (Clostridium cluster XIVa), the Clostridium leptum subgroup (Clostridium cluster IV) and the Bacteroides fragilis group had significantly increased in PHGG-fed mice. On analysis of SCFA, we found that the caecal content of acetic acid, propionic acid and butyric acid had significantly increased in PHGG-fed mice. Together, these results suggest that chronic ingestion of PHGG prevents the development of TNBS-induced colitis in mice by modulating the intestinal microbiota and SCFA, which may be significant in the development of therapeutics for inflammatory bowel disease.
\end{abstract}

Key words: Partially hydrolysed guar gum: 2,4,6-Trinitrobenzene sulfonic acid-induced colitis: Microbiota: SCFA

Inflammatory bowel diseases (IBD), including Crohn's disease (CD) and ulcerative colitis (UC), are characterised by chronic inflammation of the gastrointestinal tract. The incidence of IBD is greater in developed countries, such as Western countries; however, its incidence in East Asian countries such as Japan, Korea and China is also increasing ${ }^{(1,2)}$. The aetiology of IBD most likely involves a complex interaction of genetic, environmental and immunoregulatory factors, but its precise pathogenesis remains unclear ${ }^{(3)}$. Dietary factors, such as an increased prevalence of the 'Western' diet, which is high in fat and protein but low in fruits and vegetables, may be associated with the increase in the number of patients with IBD. Hou et al. ${ }^{(4)}$ have reported that high dietary intake of fat and meat was associated with an increased risk for IBD, and high fibre, fruit and vegetable intake was associated with a decreased risk for IBD.
Thus, dietary fibre might be involved in the pathogenesis of IBD and might be an important constituent of a therapeutic strategy for IBD, although low-fibre diets are frequently recommended for IBD patients with strictures ${ }^{(5)}$.

Partially hydrolysed guar gum (PHGG) is a water-soluble dietary fibre prepared from guar gum and used as a food stabiliser and thickener ${ }^{(6)}$. PHGG has been reported to improve symptoms associated with both constipation- and diarrhoeapredominant forms of irritable bowel syndrome ${ }^{(7)}$. In addition, dietary PHGG has been demonstrated to be beneficial in the treatment of cholera ${ }^{(8)}$, small intestinal bacterial overgrowth ${ }^{(9)}$, paediatric functional gastrointestinal disorders ${ }^{(10)}$ and the metabolic syndrome-related functions such as aberrant lipid and glucose metabolism ${ }^{(11,12)}$. With regard to intestinal inflammation, PHGG treatment has been shown to significantly

Abbreviations: IBD, inflammatory bowel diseases; MPO, myeloperoxidase; PHGG, partially hydrolysed guar gum; TNBS, 2,4,6-trinitrobenzene sulfonic acid.

* Corresponding author: T. Takagi, fax +81 75251 0710, email takatomo@koto.kpu-m.ac.jp 
reduce colonic mucosal damage in a dextran sulphate sodiuminduced colitis model, a widely accepted murine model for $\mathrm{UC}^{(13)}$

PHGG, similar to other dietary fibres, is not digested in the upper gastrointestinal tract and is fermented by colonic bacteria, resulting in the formation of SCFA, particularly butyrate. Ohashi et $a l .{ }^{(14)}$ have demonstrated that PHGG consumption stimulated the growth of butyrate-producing bacteria and, interestingly, Bifidobacterium in the intestine. Therefore, PHGG should have beneficial effects on host health through the alteration of colonic microbiota and production of SCFA. However, the mechanisms underlying the alteration of colonic microbiota and production of colonic SCFA after PHGG treatment have not been fully investigated.

In this study, we quantified selected bacterial taxa and SCFA composition in murine caecal faeces after PHGG administration to study the effects of PHGG on colonic mucosal inflammation in a murine 2,4,6-trinitrobenzene sulfonic acid (TNBS)-induced colitis model, which shares immunological and pathological features with human CD.

\section{Methods}

\section{Animals}

Male C57BL/6 mice ( $n$ 48) aged 7 weeks were obtained from SHIMIZU Laboratory Supplies Co. Ltd. The mice were caged individually in a room kept at $18-24^{\circ} \mathrm{C}$ and $40-70 \%$ relative humidity, with a $12 \mathrm{~h}$ light- $12 \mathrm{~h}$ dark cycle and ad libitum access to food and drinking water. All animals were randomised into groups receiving either a PHGG or an American Institute of Nutrition (AIN)-93G diet (Nihon Clea). The animals receiving a PHGG diet were exposed to 5\% PHGG for 2 weeks before the induction of colitis with TNBS. All animals were maintained and experimental procedures carried out in accordance with National Institutes of Health (NIH) guidelines for the use of experimental animals. All experimental protocols were approved by the Animal Care Committee of Kyoto Prefectural University of Medicine.

\section{Induction of colitis}

To induce colitis, $100 \mathrm{mg} / \mathrm{kg}$ TNBS (Sigma-Aldrich Japan) dissolved in 30\% ethanol was intrarectally administered to lightly anaesthetised (ketamine/xylazine) mice via a catheter. The control mice were intrarectally administered with $30 \%$ ethanol. A duration of $3 \mathrm{~d}$ after TNBS administration, the animals were killed, and the colon was removed and examined. The severity of colonic damage was graded according to previously established criteria $^{(15,16)}$. The presence of visible damage, serosal adhesions, diarrhoea, strictures and bowel wall thickening was included in the macroscopic grading. All scoring was performed by the same individual under blinded conditions to prevent observer bias.

\section{Histologic evaluation}

Specimens of the distal colon were fixed in $10 \%$ neutral buffered formalin. The severity of histological damage was graded according to previously established criteria ${ }^{(17)}$. In brief, the histological scores represented the presence and extent of inflammation, damage, and regeneration in colitis. After fixation, the specimens were embedded in paraffin, divided into $4-\mu \mathrm{m}$ sections, and stained with haematoxylin-eosin.

\section{Measurement of myeloperoxidase activity}

Tissue-associated myeloperoxidase (MPO) activity was measured as an index of neutrophil accumulation in the colonic mucosa by a modification of the method of Grisham et $a l^{(18)}$. The colonic mucosa (4-cm length of distal colon) was scraped off using two glass slides and was homogenised with $1.0 \mathrm{ml}$ of PBS in a Teflon Potter-Elvehjem homogenizer (As One Corp.). The resultant mucosal homogenates were centrifuged at $20000 \boldsymbol{g}$ for $15 \mathrm{~min}$ at $4^{\circ} \mathrm{C}$ to separate out the insoluble cellular debris. The pellet was rehomogenised in $0.3 \mathrm{ml}$ of $0.05 \mathrm{~mol}$ potassium phosphate buffer ( $\mathrm{pH} 5.4$ ) containing $0.5 \%$ hexadecyltrimethylammonium bromide. The samples were centrifuged at $20000 \boldsymbol{g}$ for $15 \mathrm{~min}$ at $4{ }^{\circ} \mathrm{C}$, and the supernatants were saved. MPO activity was assessed by measuring the $\mathrm{H}_{2} \mathrm{O}_{2}$-dependent oxidation of 3,3',5,5'tetramethylbenzidine. One unit of enzyme activity was defined as the amount of MPO that caused a change in the $1.0 / \mathrm{min}$ absorbance at $460 \mathrm{~nm}$ and $37^{\circ} \mathrm{C}$. The total protein in the tissue homogenates was measured with a Bio-Rad Protein Assay kit (Bio-Rad Laboratories) according to the manufacturer's protocol.

\section{Measurement of the intestinal TNF- $\alpha$ mRNA expression}

The colonic mRNA expression of $T N F-\alpha$ and $\beta$-actin (internal control) was determined by real-time PCR according to a previously used protocol ${ }^{(19)}$. Total RNA was isolated using the acid guanidinium-phenol-chloroform method with an Isogen kit (Nippon Gene Co. Ltd). The isolated RNA was stored at $-80^{\circ} \mathrm{C}$ until use in real-time PCR. Later, $1 \mu \mathrm{g}$ of extracted RNA was reverse-transcribed into first-strand complementary DNA (cDNA) using High Capacity cDNA Reverse Transcription Kit (Applied Biosystems). Real-time PCR for TNF- $\alpha$ and $\beta$-actin was conducted with a 7300 Real-Time PCR system (Applied Biosystems) using the DNA-binding dye SYBR Green to detect the PCR products, using the following primers: for $T N F-\alpha$, sense 5'-ATCCGCGACGTGGAACTG-3' and antisense 5'-ACCGCC TGGAGTTCTGGAA-3'; and for $\beta$-actin, sense 5'-TATCCA CCTTCCAGCAGATGT-3' and antisense 5'-AGCTCAGTAAC AGTCCGCCTA-3'. mRNA expression was quantified relative to $\beta$-actin expression.

\section{Determination of the intestinal TNF- $\alpha$ content}

We determined the concentration of TNF- $\alpha$ in the supernatant of mucosal homogenates using an ELISA kit (eBioscience Inc.), specific for mouse TNF- $\alpha$, following a sandwich method. The assay was performed according to the manufacturer's instructions. After colour development, optimal densities were measured at $450 \mathrm{~nm}$ with a microplate reader (Spectramax M2; Molecular Devices Corp.).

\section{Analysis of caecal bacteria}

Bacterial genomic DNA was extracted from caecal contents (approximately $50 \mathrm{mg}$ ), according to the method described by 
Godon et al. $^{(20)}$. The $16 \mathrm{~S}$ rRNA gene of the Clostridium coccoides group (Clostridium cluster XIVa), Clostridium leptum subgroup (Clostridium cluster IV), Clostridium cluster I, Clostridium cluster XI, Bacteroides fragilis group, Enterococcus, Lactobacillus and Enterobacteriaceae was quantified by realtime PCR performed with an MyiQ real-time PCR system (Bio-Rad). The reaction mixture $(20 \mu \mathrm{l})$ contained $10 \mu \mathrm{l}$ of the iQ SYBER Green Supermix (Bio-Rad), $0.5 \mu \mathrm{l}$ of caecal DNA and $400 \mu \mathrm{mol} / 1$ of each primer. Previously described primers were used in this study ${ }^{(21-23)}$. The thermal programme consisted of initial denaturation at $95^{\circ} \mathrm{C}$ for $3 \mathrm{~min}$, followed by forty cycles of denaturation at $94^{\circ} \mathrm{C}$ for $10 \mathrm{~s}$, annealing at optimum temperature for $30 \mathrm{~s}$, elongation at $72^{\circ} \mathrm{C}$ for $40 \mathrm{~s}$, and final elongation at $72^{\circ} \mathrm{C}$ for $5 \mathrm{~min}$. Fluorescent products were detected at the last step of each cycle. Melting curve analysis of the product was performed after amplification to determine the specificity of the PCR. A plasmid containing a partial sequence of the $16 \mathrm{~S}$ rRNA gene identical to that of the targeted bacteria was constructed in our laboratory and used as standard DNA.

\section{Analysis of faecal organic acids}

A portion of the caecal contents $(0.5 \mathrm{~g})$ was suspended with $0.5 \mathrm{ml}$ of $14 \%$ perchloric acid to eliminate proteins. After centrifugation at $10000 \mathrm{~g}$ at $4^{\circ} \mathrm{C}$ for $5 \mathrm{~min}$, the supernatant was filtered through a cellulose-acetate membrane filter with $0 \cdot 45-\mu \mathrm{m}$ pores, and its organic acid content was analysed by ion-exclusion HPLC as described by Ushida \& Sakata ${ }^{(24)}$.

\section{Statistical analysis}

Results are presented as means with their standard errors. Overall differences between groups were determined by one-way ANOVA. If the results of the one-way ANOVA were significant, differences between individual groups were analysed by the Bonferroni multiple comparison test. Histological scores were statistically analysed using the Mann-Whitney $U$ test. For analysis of caecal bacteria, the copy number of targeted genes and concentration of organic acids were analysed using the Friedman test, followed by the Steel-Dwass method. Values of $P<0.05$ were considered significant. All analyses were performed using the GraphPad Prism 6 program (GraphPad Software Inc.) for Macintosh.

\section{Results}

Effect of partially hydrolysed guar gum on trinitrobenzene sulfonic acid-induced colonic damage

Macroscopic findings in the colon of mice exposed to TNBS indicated severe colitis with hyperaemia, oedema, thickening and ulceration. Pre-feeding treatment with 5\% PHGG reduced the size of the macroscopic lesion (Fig. 1(a)). The colonic damage score had increased significantly after TNBS administration, but this increase was significantly dampened by $5 \%$ PHGG treatment (Fig. 1(b)). In addition, the degree of body weight loss after the induction of colitis with TNBS was significantly inhibited in 5\% PHGG-treated mice (Fig. 1(c)).

Fig. 2(a) shows typical histological features in the TNBS-only and 5\% PHGG-treated groups. Haematoxylin-eosin-stained (a)

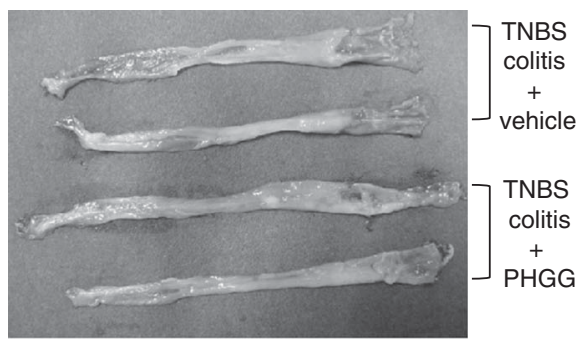

(c)

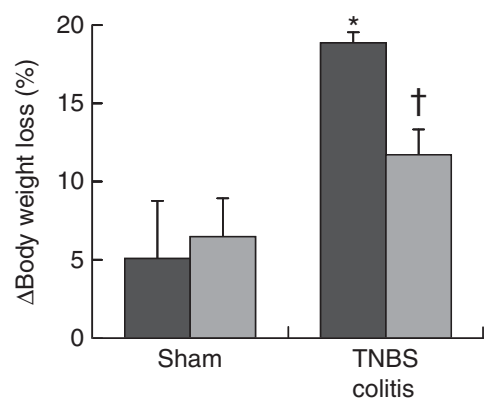

(b)

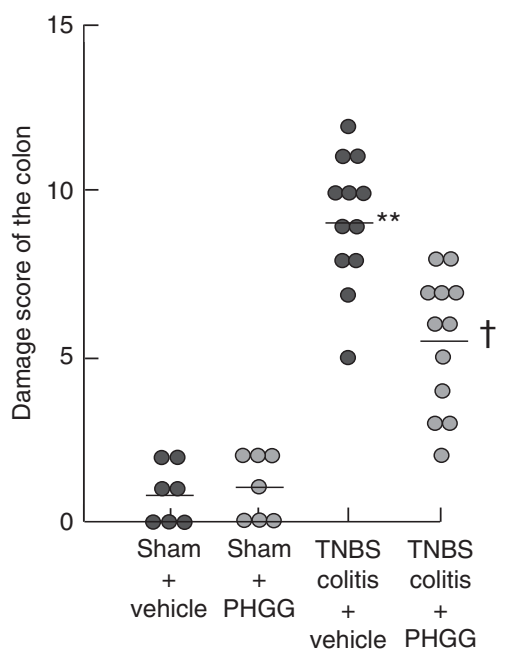

Fig. 1. Effects of partially hydrolysed guar gum (PHGG) on 2,4,6-trinitrobenzene sulfonic acid (TNBS)-induced colonic damage. (a) Representative macroscopic findings in the colon on day 3 after the induction of injury by TNBS administration. TNBS induced severe colitis with hyperaemia, oedema, thickening, ulceration and necrosis, which were reduced in mice treated with PHGG. (b) Damage score of the colon of mice treated with PHGG until day 3 after the induction of colitis. Data are expressed as a scatter plot. ${ }^{* \star} P<0.01$ compared with the sham group. $\dagger P<0.05$ compared with the TNBS colitis group. (c) Decrease in body weight after the induction of TNBS colitis was significantly attenuated in mice treated with PHGG. ${ }^{*} P<0.05$ compared with the sham group. $\dagger P<0.05$ compared with the TNBS-induced colitis group. $\square$, PHGG; $\square$, TNBS. 
(a)

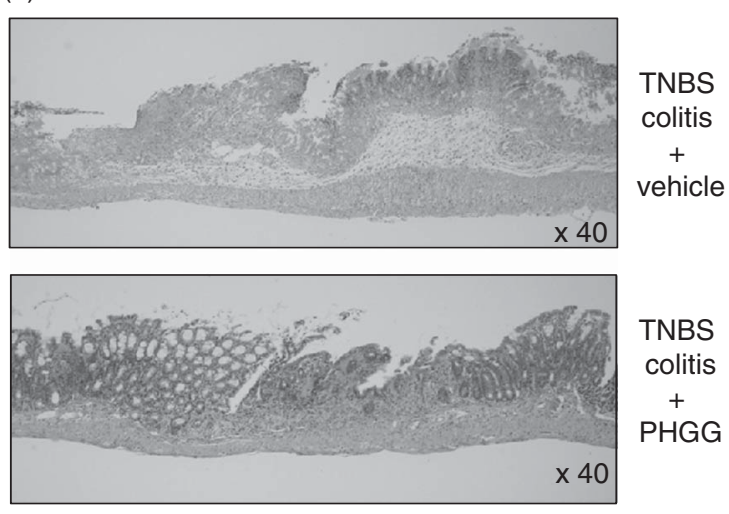

(b)

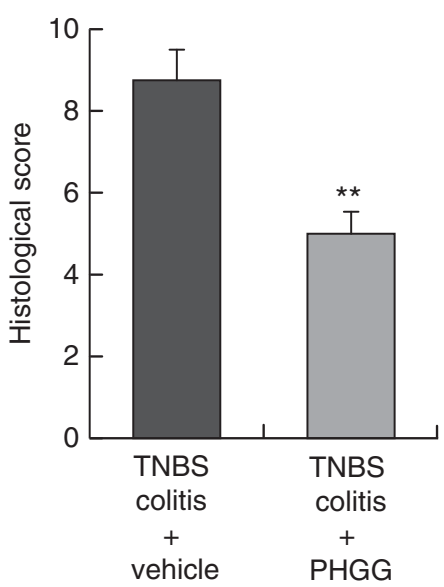

(c)

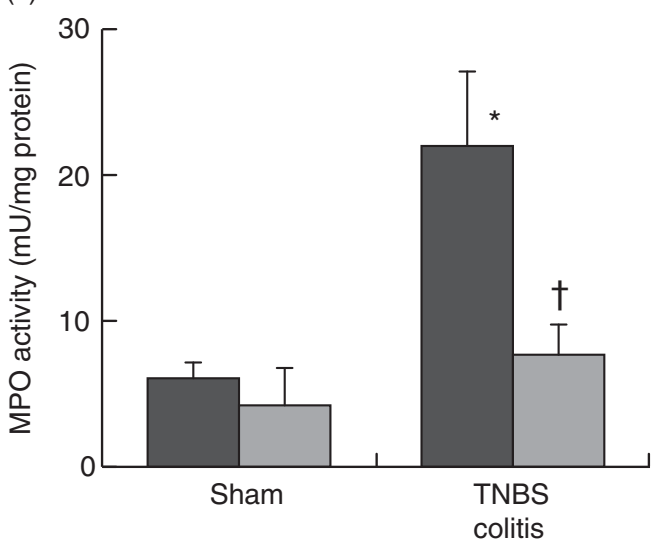

Fig. 2. Effects of partially hydrolysed guar gum (PHGG) on 2,4,6-trinitrobenzene sulfonic acid (TNBS)-induced colitis. (a) Histological appearance of the colon of mice administered only TNBS and of those treated with PHGG and administered TNBS. Magnification, 40x. Haematoxylin-eosin staining. (b) Histological score of the colon of mice administered only TNBS and of those treated with PHGG and administered TNBS. Values are means $(n 5)$, with their standard errors. ${ }^{* \star} P<0.01$ compared with vehicle-treated mice with TNBS-induced colitis. (c) Tissue-associated myeloperoxidase (MPO) activity as an index of neutrophil accumulation in the colonic mucosa. Values are means $(n 7)$, with their standard errors. ${ }^{*} P<0.05$ compared with the sham group. $\dagger P<0.05$ compared with the TNBS colitis group. $\square$, Vehicle; $\square$, PHGG.

colonic specimens of TNBS-only mice showed extensive ulcer formation with massive infiltration of inflammatory cells, which were predominantly neutrophils. In contrast, specimens of $5 \%$ PHGG-treated mice showed lesser ulceration and infiltration of inflammatory cells. Further, the histological scores of $5 \%$ PHGG-treated mice were significantly lower than those of TNBS-only mice (Fig. 2(b)).

\section{Effects of partially hydrolysed guar gum treatment on myeloperoxidase activity}

Neutrophil accumulation was evaluated by measuring tissueassociated MPO activity in colonic mucosal homogenates. In the sham groups, there were no differences in the MPO activities of vehicle- and 5\% PHGG-treated mice. However, in the TNBS-treated groups, MPO activity in the colonic mucosa was markedly increased after TNBS administration compared with the sham groups. This increase after TNBS administration was significantly inhibited by treatment with 5\% PHGG (Fig. 2(c)).

\section{Effect of partially hydrolysed guar gum treatment on TNF- $\alpha$ expression}

To investigate the effect of PHGG treatment on TNF- $\alpha$ expression in the colonic mucosa, we analysed the colonic TNF- $\alpha$ mRNA expression and mucosal TNF- $\alpha$ protein content using ELISA. In the sham-operated groups, there were no significant differences between the vehicle- and 5\% PHGG-treated mice. However, the colonic expression of $T N F-\alpha$ mRNA was significantly increased in TNBS-treated mice, and this increase was significantly reduced by 5\% PHGG treatment (Fig. 3(a)). Similarly, the mucosal TNF- $\alpha$ protein content was significantly increased in TNBS-treated mice, and this increase was significantly attenuated by 5\% PHGG treatment (Fig. 3(b)).

\section{Effect of partially hydrolysed guar gum on the caecal bacterial profile}

To determine whether PHGG treatment altered the caecal bacterial profile, we examined the variation in eight bacterial groups using real-time PCR. As shown in Table 1, compared 
(a)

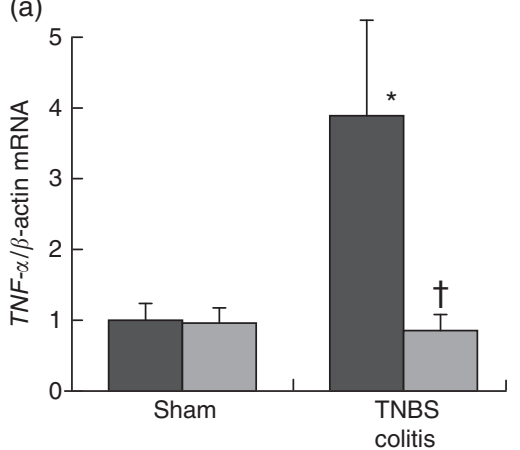

(b)

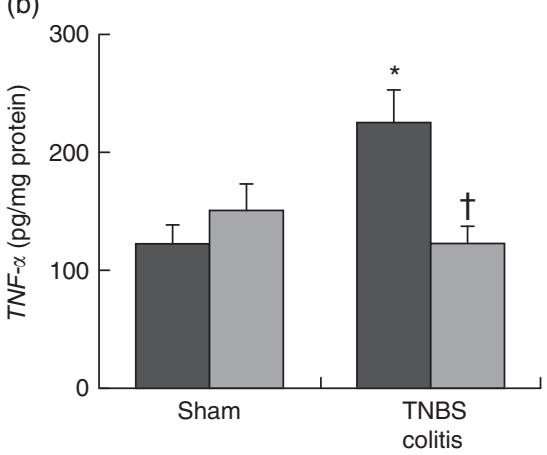

Fig. 3. Effect of partially hydrolysed guar gum (PHGG) on TNF- $a$ expression in the colonic mucosa after TNBS-induced injury. (a) TNF- $a$ mRNA expression in colonic mucosa. Values are means $(n 7)$, with their standard errors. ${ }^{*} P<0.05$ compared with the sham group. $\dagger P<0.05$ compared with the TNBS colitis group. (b) Concentration of TNF- $a$ in the supernatant of mucosal homogenates. Values are means $(n 7)$, with their standard errors. ${ }^{*} P<0.05$ compared with the sham group. $\dagger P<0.05$ compared with the TNBS colitis group. $\square$, Vehicle; $\square$, PHGG.

Table 1. The alteration of colonic microbiota after treatment with $5 \%$ partially hydrolysed guar gum (PHGG) (Mean values and standard deviations, seven mice per group)

\begin{tabular}{|c|c|c|c|c|}
\hline & \multicolumn{2}{|c|}{ Control diet (AIN-93G) log (copy/g caecal contents) } & \multicolumn{2}{|c|}{$5 \%$ PHGG log (copy/g caecal contents) } \\
\hline & Mean & SD & Mean & SD \\
\hline Clostridium coccoides group & 11.42 & 0.078 & 11.80 & $0.309^{\star \star}$ \\
\hline Clostridium leptum subgroup & $10 \cdot 18$ & 0.270 & 10.58 & $0.380^{*}$ \\
\hline Clostridium cluster I & $10 \cdot 33$ & 0.197 & $10 \cdot 16$ & 0.584 \\
\hline Clostridium cluster XI & 9.808 & 0.268 & 8.259 & $0.292^{*}$ \\
\hline Bacteroides fragilis group & $12 \cdot 01$ & 0.423 & $12 \cdot 81$ & $0.271^{\star \star}$ \\
\hline Lactobacillus group & 9.724 & 0.395 & 9.843 & 0.702 \\
\hline Enterococcus & 9.072 & $1 \cdot 144$ & 9.067 & 0.867 \\
\hline Enterobacteriaceae & $10 \cdot 23$ & 1.796 & $10 \cdot 75$ & 0.885 \\
\hline
\end{tabular}

${ }^{*} P<0.05,{ }^{* *} P<0.01$ v. control diet (AIN-93G).

Table 2. The alteration of SCFA in the colonic lumen after treatment with $5 \%$ partially hydrolysed guar gum (PHGG) (Mean values and standard deviations, seven mice per group)

\begin{tabular}{|c|c|c|c|c|}
\hline & \multicolumn{2}{|c|}{ Control diet (AIN-93G) $(\mu \mathrm{mol} / \mathrm{g}$ caecal contents) } & \multicolumn{2}{|c|}{$5 \%$ PHGG ( $\mu \mathrm{mol} / \mathrm{g}$ caecal contents) } \\
\hline & Mean & SD & Mean & SD \\
\hline Succinic acid & 0.41 & 0.33 & $2 \cdot 89$ & $1 \cdot 34^{\star \star}$ \\
\hline Lactic acid & 0.05 & $0 \cdot 12$ & 0.66 & 1.01 \\
\hline Formic acid & 1.23 & 0.29 & 1.27 & 0.53 \\
\hline Acetic acid & 21.5 & 6.29 & $32 \cdot 6$ & $10 \cdot 2^{*}$ \\
\hline Propionic acid & $6 \cdot 13$ & 0.74 & $10 \cdot 8$ & $2 \cdot 10^{\star \star}$ \\
\hline Isobutyric acid & 0.73 & 0.15 & 0.58 & $0 \cdot 16$ \\
\hline Butyric acid & $1 \cdot 70$ & 1.59 & 3.55 & $2 \cdot 54^{*}$ \\
\hline Isovaleric acid & 0.54 & 0.08 & 0.40 & 0.20 \\
\hline Valeric acid & 0.64 & 0.08 & 0.48 & 0.16 \\
\hline
\end{tabular}

${ }^{*} P<0.05,{ }^{* *} P<0.01$ v. control diet (AIN-93G).

with vehicle-treated mice, 5\% PHGG-treated mice showed significantly increased caecal proliferation of the $C$. coccoides group (Clostridium cluster XIVa), the $C$. leptum subgroup (Clostridium cluster IV) and the B. fragilis group. Conversely, $5 \%$ PHGG treatment significantly reduced the proliferation of caecal Clostridium cluster XI compared with control diet.

\section{Effect of partially hydrolysed guar gum on the caecal SCFA profile}

To determine whether PHGG treatment altered the caecal SCFA profile, we determined the caecal SCFA content by using ion-exclusion HPLC (Table 2). PHGG treatment stimulated the production of acetic acid, propionic acid, butyric acid and succinic acid compared with the control diet. No differences were observed in the production of formic acid, lactic acid, isobutyric acid, isovaleric acid and valeric acid.

\section{Discussion}

We demonstrated that treatment with PHGG ameliorated TNBSinduced colonic injury and inflammation in mice. In our study, colonic injury was assessed on the basis of the macroscopic damage and histological scores, and treatment with PHGG was 
found to significantly reduce colonic injury. In addition, we showed that the MPO activity and TNF- $\alpha$ mRNA and protein production in the intestinal mucosa were enhanced in TNBS-induced intestinal inflammation, and these increases were significantly reversed by PHGG treatment.

TNF- $\alpha$ has recently attracted attention for its key role in the pathogenesis of IBD, and TNF- $\alpha$-blocking agents have been used as therapeutic agents for treating IBD worldwide ${ }^{(25)}$. Our results are consistent with those of a previous study that used a different murine colitis model to demonstrate that PHGG ameliorated murine colonic inflammation through inhibition of colonic mucosal TNF- $\alpha$ production ${ }^{(13)}$. Although the detailed mechanism by which PHGG may inhibit TNF- $\alpha$ production remains to be elucidated, PHGG may have therapeutic potential for patients with IBD.

An important result of the present study is that the alerted profiles of caecal bacteria and SCFA can be identified after PHGG treatment. Recent studies have demonstrated that microbiota plays a role in the onset and progression of intestinal inflammation in IBD, and the therapeutic efficacy of antibiotics, probiotics and prebiotics in relation to their effects on microbiota has been noted in the treatment of $\mathrm{IBD}^{(26)}$. With regard to the caecal bacterial profile, we classified the bacteria into the C. coccoides group (Clostridium cluster XIVa), the C. leptum subgroup (Clostridium cluster IV) and the B. fragilis group as SCFA-producing indigenous bacteria, Enterococcus as indigenous bacteria, Clostridium cluster I and XI, which contains wellknown harmful bacteria including Clostridium difficile and Clostridium perfringens, the Lactobacillus group as lactateproducing bacterium, and Enterobacteriaceae as pathogenic bacteria, such as Escherichia coli. PHGG treatment significantly increased the concentration of the caecal $C$. coccoides group (Clostridium cluster XIVa), the C. leptum subgroup (Clostridium cluster IV), and the $B$. fragilis group and significantly decreased the concentration of caecal Clostridium cluster XI compared with the control diet.

Interestingly, Sokol et al. ${ }^{(27)}$ have shown that the C. leptum subgroup and $C$. coccoides group were less represented in patients with active IBD. Moreover, Ando et al. ${ }^{(28)}$ have demonstrated that Clostridium cluster XIVa and IV were significantly decreased in patients with active-phase CD than in healthy individuals. Thus, a reduction in these bacteria may be associated with susceptibility to intestinal inflammation. Therefore, an increase in these bacteria by PHGG administration might lead to protective effects against intestinal inflammation. Koleva et al. ${ }^{(29)}$ have reported that chronic intestinal inflammation consistently correlated with the abundance of Clostridium cluster XI, which are well-known harmful bacteria, in HLA-B27 transgenic rats, a well-validated rodent model for IBD, and they focused on reducing Clostridium cluster XI to inhibit intestinal inflammation by treatment with inulin and fructo-oligosaccharides. In agreement with these data, in the present study, PHGG administration reduced Clostridium cluster XI, which correlated with a decrease in chronic intestinal inflammation.

Compositional alterations of the colonic microbiota may also change metabolic capacities of the gut bacteria ${ }^{(30)}$. Colonic fermentation of carbohydrates results in the generation of SCFA, which are generally assumed to be beneficial for the host ${ }^{(31)}$. Reduced levels of faecal SCFA have previously been linked to a shift in the composition and metabolic activity of the colonic microbiota in $\operatorname{IBD}^{(27,28)}$. Treatment with PHGG caused a significant increase in the caecal content of the SCFA-producing bacterial group, the C. coccoides group (Clostridium cluster $\mathrm{XIV}$ ), the C. leptum group (Clostridium cluster IV) and the $B$. fragilis group. These results were consistent with those of previous experiments that indicated that PHGG stimulated butyrate-producing bacteria $^{(14)}$. Recent reports have demonstrated that Clostridium cluster XIV, Clostridium cluster IV, and the $B$. fragilis group promoted accumulation of $\mathrm{CD}^{+} \mathrm{T}$ regulatory $\left(\mathrm{T}_{\mathrm{reg}}\right)$ cells ${ }^{(32,33)}$. In addition, Smith et al. ${ }^{(34)}$ showed that three SCFA (propionate, acetate and butyrate), individually or in combination, derived from common bacterial metabolites increased the number of $\mathrm{T}_{\text {reg }}$ cells in the large intestine. In our study, production of acetic acid, propionic acid and butyric acid was stimulated by PHGG treatment. The inhibition of TNBSinduced colitis by PHGG treatment might be due to the promotion of $\mathrm{T}_{\text {reg }}$ cells, which are critical for limiting intestinal inflammation ${ }^{(35)}$; however, we could not fully investigate this issue in this study. Thus, dietary fibre stimulates the growth of intestinal bacteria such as Clostridium cluster XIV, Clostridium cluster IV, and the B. fragilis group and may enhance the microbial balance in the colon, because these bacteria produce SCFA and anti-microbial compounds that may protect against increased growth of potentially pathogenic bacteria such as C. perfringens and E. coli ${ }^{(36)}$.

Our study includes potential limitations. Our investigation evaluated a limited list in intestinal microbiota and did not investigate several important bacteria including Bifidobacterium. In addition, we evaluated SCFA and microbiota after the development of TNBS-induced colitis. Therefore, further experiments would be required to elucidate these issues.

In summary, our results indicate that PHGG treatment inhibits intestinal inflammation in mice, which is associated with a significant decrease in TNF- $\alpha$ production and neutrophil infiltration in the intestinal mucosa. These effects may lead to changes in caecal bacterial and SCFA profiles. Although further studies are warranted to unravel the mechanisms underlying this phenomenon, the administration of PHGG has potential as a new therapeutic strategy for IBD.

\section{Acknowledgements}

This work was supported by Grants-in-Aid for Scientific Research (KAKENHI) (C) to T. T. (no. 25460959) and Y. N. (no. 25460958) from the Japan Society for the Promotion of Science and by an Adaptable and Seamless Technology Transfer Program Through Target-Driven R\&D (to Y. N.) grant from the Japan Agency for Medical Research and Development.

T. T. and Y. N. conceived this experiments; T. T., Y. H., C. U., K. M., K. Katada and K. Kamada performed the majority of in vivo experiments; K. U. and O. H. performed the majority of TNF expression experiments; Y. O. performed analysis of caecal bacteria; Z. Y., M. O., M. T. and T. O. performed analysis of faecal organic acids; T. T., Y. I. and T. Y. analysed data and 
were also involved in editing the manuscript. All authors discussed the results and commented on the manuscript.

Y. N. received scholarship funds from Eisai Co. Ltd, Astellas Pharma Inc., Takeda Pharmaceutical Co. Ltd and Mitsubishi Tanabe Pharma Co. Ltd. The other authors have no conflicts of interest to disclose.

\section{References}

1. Asakura K, Nishiwaki Y, Inoue N, et al. (2009) Prevalence of ulcerative colitis and Crohn's disease in Japan. J Gastroenterol 44, 659-665.

2. Molodecky NA, Soon IS, Rabi DM, et al. (2012) Increasing incidence and prevalence of the inflammatory bowel diseases with time, based on systematic review. Gastroenterology 142 , 46-54.e42; quiz e30.

3. Xavier RJ \& Podolsky DK (2007) Unravelling the pathogenesis of inflammatory bowel disease. Nature 448, 427-434.

4. Hou JK, Abraham B \& El-Serag H (2011) Dietary intake and risk of developing inflammatory bowel disease: a systematic review of the literature. Am J Gastroenterol 106, 563-573.

5. Lewis JD \& Fisher RL (1994) Nutrition support in inflammatory bowel disease. Med Clin North Am 78, 1443-1456.

6. Yoon SJ, Chu DC \& Raj Juneja L (2008) Chemical and physical properties, safety and application of partially hydrolized guar gum as dietary fiber. J Clin Biochem Nutr 42, 1-7.

7. Giannini EG, Mansi C, Dulbecco P, et al. (2006) Role of partially hydrolyzed guar gum in the treatment of irritable bowel syndrome. Nutrition 22, 334-342.

8. Alam NH, Ashraf H, Sarker SA, et al. (2008) Efficacy of partially hydrolyzed guar gum-added oral rehydration solution in the treatment of severe cholera in adults. Digestion 78, 24-29.

9. Furnari M, Parodi A, Gemignani L, et al. (2010) Clinical trial: the combination of rifaximin with partially hydrolysed guar gum is more effective than rifaximin alone in eradicating small intestinal bacterial overgrowth. Aliment Pharmacol Ther 32, 1000-1006.

10. Romano C, Comito D, Famiani A, et al. (2013) Partially hydrolyzed guar gum in pediatric functional abdominal pain. World J Gastroenterol 19, 235-240.

11. Yasukawa Z, Naito Y, Takagi T, et al. (2012) Partially hydrolyzed guar gum affects the expression of genes involved in host defense functions and cholesterol absorption in colonic mucosa of $\mathrm{db} / \mathrm{db}$ male mice. J Clin Biochem Nutr 51, 33-38.

12. Dall'Alba V, Silva FM, Antonio JP, et al. (2013) Improvement of the metabolic syndrome profile by soluble fibre - guar gum - in patients with type 2 diabetes: a randomised clinical trial. Br J Nutr 110, 1601-1610.

13. Naito Y, Takagi T, Katada K, et al. (2006) Partially hydrolyzed guar gum down-regulates colonic inflammatory response in dextran sulfate sodium-induced colitis in mice. J Nutr Biochem 17, 402-409.

14. Ohashi Y, Sumitani K, Tokunaga M, et al. (2015) Consumption of partially hydrolysed guar gum stimulates bifidobacteria and butyrate-producing bacteria in the human large intestine. Benef Microbes 6, 451-455.

15. Harusato A, Naito Y, Takagi T, et al. (2013) BTB and CNC homolog 1 (Bach1) deficiency ameliorates TNBS colitis in mice: role of M2 macrophages and heme oxygenase- 1 . Inflamm Bowel Dis 19, 740-753.

16. Takagi T, Naito Y, Mizushima K, et al. (2010) Inhalation of carbon monoxide ameliorates TNBS-induced colitis in mice through the inhibition of TNF-alpha expression. Dig Dis Sci 55, 2797-2804.
17. Elson CO, Beagley KW, Sharmanov AT, et al. (1996) Hapteninduced model of murine inflammatory bowel disease: mucosa immune responses and protection by tolerance. J Immunol 157, 2174-2185.

18. Grisham MB, Hernandez LA \& Granger DN (1986) Xanthine oxidase and neutrophil infiltration in intestinal ischemia. $\mathrm{Am} \mathrm{J}$ Physiol 251, G567-G574.

19. Higashimura Y, Naito Y, Takagi T, et al. (2013) Oligosaccharides from agar inhibit murine intestinal inflammation through the induction of heme oxygenase-1 expression. J Gastroenterol 48, 897-909.

20. Godon JJ, Zumstein E, Dabert P, et al. (1997) Molecular microbial diversity of an anaerobic digestor as determined by small-subunit rDNA sequence analysis. Appl Environ Microbiol 63, 2802-2813.

21. Song Y, Liu C \& Finegold SM (2004) Real-time PCR quantitation of clostridia in feces of autistic children. Appl Environ Microbiol 70, 6459-6465.

22. Rinttila T, Kassinen A, Malinen E, et al. (2004) Development of an extensive set of 16S rDNA-targeted primers for quantification of pathogenic and indigenous bacteria in faecal samples by real-time PCR. J Appl Microbiol 97, 1166-1177.

23. Matsuki T, Watanabe K, Fujimoto J, et al. (2004) Use of $16 \mathrm{~S}$ rRNA gene-targeted group-specific primers for real-time PCR analysis of predominant bacteria in human feces. Appl Environ Microbiol 70, 7220-7228.

24. Ushida K \& Sakata T (1998) Effect of pH on oligosaccharide fermentation by porcine cecal digesta. Anim Sci Tech 69, 100-107.

25. Rutgeerts P, Vermeire S \& Van Assche G (2009) Biological therapies for inflammatory bowel diseases. Gastroenterology 136, 1182-1197.

26. Sartor RB (2004) Therapeutic manipulation of the enteric microflora in inflammatory bowel diseases: antibiotics, probiotics, and prebiotics. Gastroenterology 126, 1620-1633.

27. Sokol H, Seksik P, Furet JP, et al. (2009) Low counts of Faecalibacterium prausnitzii in colitis microbiota. Inflamm Bowel Dis 15, 1183-1189.

28. Andoh A, Kuzuoka H, Tsujikawa T, et al. (2012) Multicenter analysis of fecal microbiota profiles in Japanese patients with Crohn's disease. J Gastroenterol 47, 1298-1307.

29. Koleva PT, Valcheva RS, Sun X, et al. (2012) Inulin and fructooligosaccharides have divergent effects on colitis and commensal microbiota in HLA-B27 transgenic rats. Br J Nutr 108, $1633-1643$

30. De Preter V, Machiels K, Joossens M, et al. (2015) Faecal metabolite profiling identifies medium-chain fatty acids as discriminating compounds in IBD. Gut 64, 447-458.

31. Macfarlane GT \& Macfarlane S. (2011) Fermentation in the human large intestine: its physiologic consequences and the potential contribution of prebiotics. J Clin Gastroenterol $\mathbf{4 5}$, S120-S127.

32. Atarashi $\mathrm{K}$, Tanoue $\mathrm{T}$, Shima $\mathrm{T}$, et al. (2011) Induction of colonic regulatory $\mathrm{T}$ cells by indigenous Clostridium species. Science 331, 337-341.

33. Round JL \& Mazmanian SK (2010) Inducible Foxp3+ regulatory T-cell development by a commensal bacterium of the intestinal microbiota. Proc Natl Acad Sci U S A 107, 12204-12209.

34. Smith PM, Howitt MR, Panikov N, et al. (2013) The microbial metabolites, short-chain fatty acids, regulate colonic Treg cell homeostasis. Science 341, 569-573.

35. Atarashi K, Umesaki Y \& Honda K (2011) Microbiotal influence on T cell subset development. Semin Immunol 23, 146-153.

36. Zhang YJ, Li S, Gan RY, et al. (2015) Impacts of gut bacteria on human health and diseases. Int J Mol Sci 16, 7493-7519. 